\title{
Efecto de diferentes densidades de cría sobre el crecimiento y la supervivencia de Rhamdia quelen juveniles (Pisces, Siluriformes)
}

\author{
Santinón, J.J.; Hernández, D.R.; Sánchez, S.; Domitrovic, H.A. \\ Instituto de Ictiología del Nordeste, Facultad de Ciencias Veterinarias, UNNE, Sargento Cabral 2139, \\ Corrientes (3400), Argentina. Tel 03783-425753 interno 171. E-mail: jsantinon@conicet.gov.ar.
}

\begin{abstract}
Resumen
Santinón, J.J.; Hernández, D.R.; Sánchez, S.; Domitrovic, H.A.: Efecto de diferentes densidades de cría sobre el crecimiento y la supervivencia de Rhamdia quelen juveniles (Pisces, Siluriformes). Rev. vet. 23: 1, 64-68, 2012. El presente estudio fue realizado para evaluar el efecto de la densidad de cría sobre parámetros de crecimiento y supervivencia en ejemplares juveniles de Rhamdia quelen, mantenidos bajo un sistema de cría semi-intensivo. Se utilizaron 147 ejemplares con peso medio inicial de 1,27 $\pm 0,45 \mathrm{~g}$, distribuidos en nueve unidades experimentales a tres densidades de cultivo: 5,10 y 20 individuos $/ \mathrm{m}^{3}$, correspondientes a los tratamientos DI, DII y DIII, respectivamente. La experiencia tuvo una duración de 47 días, período en el cual los peces fueron alimentados con ración balanceada peletizada, conteniendo $35 \%$ de proteína. Al final de la experiencia, los individuos pertenecientes a DI presentaron coeficientes de crecimiento específico y peso medio final significativamente superiores a los obtenidos por los individuos DIII $(\mathrm{p}<0,05)$, mientras que los pertenecientes a DII no se diferenciaron estadísticamente de los demás $(\mathrm{p}>0,05)$. Por su parte, la mayor biomasa final producida fue obtenida en DIII, diferenciándose significativamente de DI $(\mathrm{p}<0,05)$, mientras que DII no se diferenció significativamente de ninguno de los anteriores. La tasa de supervivencia fue superior al $90 \%$ en todos los grupos, sin presentar diferencias significativas entre los mismos ( $p>0,05)$. Los resultados obtenidos en el presente estudio indican que la densidad DII sería la más recomendable, alcanzando aceptables parámetros de crecimiento y altas tasas de supervivencia.
\end{abstract}

Palabras clave: Rhamdia quelen, alimentación, densidad, crecimiento, supervivencia.

\begin{abstract}
Santinón, J.J.; Hernández, D.R.; Sánchez, S.; Domitrovic, H.A.: Growth and survival of juvenile Rhamdia quelen (Pisces, Siluriformes) under different rearing densities. Rev. vet. 23: 1, 64-68, 2012. This study was conducted to assess the effect of stock density on growth and survival of juvenile Rhamdia quelen reared in a semi-intensive culture system. A total of 147 R. quelen with initial weight of $1.27 \pm 0.45 \mathrm{~g}$ were placed at densities of 5, 10 and $20 \mathrm{fish} /$ $\mathrm{m}^{3}$ (DI, DII and DIII, respectively) in nine experimental units. During a 47 days trial, fish were fed with a diet containing $35 \%$ of crude protein. Fish from DI showed a significant increase in weight gain compared to fish from DIII $(\mathrm{p}<0.05)$, while fish from DII did not differ statistically from the other treatments ( $p>0.05)$. By contrast, the highest final biomass was obtained in DIII, differing significantly from DI, while individuals from DII did not differ significantly with the other groups. Survival rate was higher than $90 \%$ in all groups, showing no significant differences between treatments $(\mathrm{p}>0.05)$. These results indicate that the middle density would be the most appropriate to obtain acceptable growth parameters and high survival rates.
\end{abstract}

Key words: Rhamdia quelen, feeding, density, growth, survival.

\section{INTRODUCCIÓN}

Dentro de las producciones no tradicionales, la piscicultura es una actividad que en la última década ganó terreno e importancia debido a los avances tecnológicos, los numerosos estudios realizados a fin de optimizar los

Recibido:16 diciembre 2011 / Aceptado: 26 enero 2012 recursos disponibles, la constante y creciente demanda de sus productos y la disminución de la producción mundial de la pesca continental y marítima ${ }^{10,19}$.

En la actualidad existe un gran número de estudios orientados a conocer y a mejorar, entre otros aspectos, los requerimientos nutricionales de los peces en diferentes categorías de crecimiento ${ }^{1,13}$, aumentar el porcentaje de supervivencia ${ }^{4,7}$, optimizar las condiciones 
físicas y químicas del agua ${ }^{3,20}$ y elaborar alimentos balanceados capaces de cubrir las necesidades alimenticias desde el inicio de la alimentación exógena ${ }^{6,7,14,21}$, permitiendo así acelerar su crecimiento y disminuir los costos de producción ${ }^{16}$.

La especie más producida en el nordeste argentino es el pacú (Piaractus mesopotamicus), sin embargo últimamente se ha puesto especial atención al bagre sudamericano (Rhamdia quelen), una especie nativa que presenta un buen potencial de cultivo por su adaptabilidad a la cría en estanques, buena calidad de carne y una amplia plasticidad térmica ${ }^{19}$, distribuyéndose geográficamente desde México hasta el sur de Argentina ${ }^{27}$.

Si bien varios estudios fueron llevados a cabo para evaluar el efecto de distintas densidades de stock en juveniles de esta especie ${ }^{2,18,19,22}$, fueron realizados bajo sistema de cría intensivo. Sin embargo, otros autores ${ }^{24}$ opinan que los estudios realizados bajo sistemas de cría semi-intensiva en esta especie aún resultan escasos. Además, esta etapa se caracteriza por presentar altas tasas de mortalidad, representando el momento de mayor dificultad del ciclo productivo ${ }^{2}$. Así, el objetivo del presente trabajo fue evaluar el efecto de tres densidades de cría sobre parámetros de crecimiento y supervivencia en juveniles de $R$. quelen, bajo un sistema de cría semi-intensivo.

\section{MATERIAL Y MÉTODOS}

El estudio fue llevado a cabo en las instalaciones de piscicultura experimental del Instituto de Ictiología del Nordeste (INICNE) de la Facultad de Ciencias Veterinarias de la UNNE (Corrientes, Argentina), abarcando un total de 47 días. Para la realización de la experiencia fueron utilizados 147 ejemplares juveniles de $R$. quelen con peso medio inicial de 1,27 $\pm 0,45 \mathrm{~g}$, distribuidos en nueve estanques de mampostería de $1,5 \mathrm{~m}^{3}$, los que fueron abastecidos con agua de perforación y aireación forzada. La renovación del agua consistió únicamente en reponer la porción de pérdida por evaporación. El diseño experimental fue completamente aleatorizado con 3 tratamientos y 3 repeticiones $(n=9)$, donde fueron evaluadas tres densidades de cría: DI, DII y DIII, correspondientes a 5, 10 y 20 individuos $/ \mathrm{m}^{3}$, respectivamente. El fondo de cada estanque fue rellenado con arena aproximadamente unos $2 \mathrm{~cm}$ de espesor para simular las condiciones ambientales encontradas normalmente en viveros de piscicultura ${ }^{8}$. Los mismos fueron fertilizados cinco días antes del inicio del ensayo con estiércol bovino a razón de $300 \mathrm{~g} / \mathrm{m}^{2} 24$. Las variables químicas del agua: nitrito, dureza, amonio, alcalinidad, conductividad y $\mathrm{pH}$, fueron medidas al inicio, a los 20 días y al finalizar el ensayo, mientras que la temperatura del agua y el oxígeno disuelto fueron registradas dos veces al día durante la totalidad de la experiencia.

Los peces fueron alimentados dos veces al día (10:00 y 18:00 h) con ración balanceada peletizada conteniendo $35 \%$ de proteína bruta ${ }^{25}$. La ración ofrecida fue previamente molida y tamizada con una malla de
Tabla 1: Valores de calidad de agua obtenidos.

\begin{tabular}{lc}
\hline parámetro & media $\pm \mathrm{DE}$ \\
\hline temperatura ${ }^{\circ} \mathrm{C}$ & $24,7 \pm 1,92$ \\
$\mathrm{pH}$ & $6,96 \pm 0,27$ \\
oxígeno $(\%)$ & $62,76 \pm 23,43$ \\
oxígeno $(\mathrm{mg} / \mathrm{l})$ & $5,05 \pm 1,95$ \\
amonio $(\mathrm{mg} / \mathrm{l})$ & $0,27 \pm 0,12$ \\
nitrito $(\mathrm{mg} / \mathrm{l})$ & $0,10 \pm 0,08$ \\
alcalinidad $(\mathrm{mg} / \mathrm{l})$ & $37,3 \pm 4,5$ \\
dureza $(\mathrm{mg} / \mathrm{l})$ & $30,22 \pm 5,76$ \\
conductividad $(\mu \mathrm{S} / \mathrm{cm})$ & $112,12 \pm 9,1$ \\
\hline
\end{tabular}

$500 \mu \mathrm{m}$ de abertura. La cantidad de alimento brindado diariamente fue equivalente al $10 \%$ de la biomasa de cada grupo durante los primeros 33 días y al 5\% el resto del ensayo hasta alcanzar los 47 días.

Durante el transcurso de la experiencia fueron realizadas mediciones biométricas cada 15 días, incluyendo en la última el recuento total de los individuos para establecer el porcentaje de supervivencia. Con los datos de peso obtenidos en las mediciones parciales fue posible calcular la biomasa media de cada estanque y corregir la cantidad de ración balanceada ofrecida a los peces diariamente.

Al finalizar el ensayo, las variables de peso medio final (PM), biomasa final (BF), coeficiente de crecimiento específico ${ }^{13}$ (CCE) y tasa de supervivencia (S) fueron calculadas de la siguiente manera: $\mathrm{PM}(\mathrm{g})=\mathrm{P}_{t}$ $N^{-1}$, donde $\mathrm{P}_{t}$ es el peso total $(\mathrm{g})$ por cada réplica y $N=$ número de individuos sobrevivientes; $\mathrm{BF}=\mathrm{PM}(N)$; $\mathrm{CCE}=\left[100\left(3\right.\right.$ (Peso final $^{0,33}-$ Peso inicial $\left.\left.\left.^{0,33}\right)(\mathrm{T})^{-1}\right)\right]$ donde $\mathrm{T}$ es la duración del ensayo en días; $\mathrm{S}(\%)=100$ $\left(N N_{i}^{-1}\right)$, donde $N_{i}=$ número inicial de peces.

La significación estadística del tratamiento fue analizada efectuándose un ANOVA a una vía. Las comparaciones de medias a posteriori se realizaron mediante el test de Tukey $(\mathrm{p}<0,05)$, efectuándose además un contraste ortogonal para determinar la significancia del efecto lineal de los niveles de densidad utilizados. Para una mejor interpretación de los resultados se realizó un análisis de regresión. Todas las pruebas estadísticas se llevaron a cabo empleando el software estadístico Statistica 6.0.

\section{RESULTADOS Y DISCUSIÓN}

Los parámetros físicos y químicos del agua se mantuvieron dentro de un rango de valores adecuados para la cría de la especie ${ }^{1}$ (Tabla 1).

Para la variable CCE (Figura 1), los individuos DI presentaron valores superiores a los observados en DIII, siendo significativamente distintos $(\mathrm{p}<0,05)$, mientras que los individuos DII no se diferenciaron significativamente de los demás tratamientos $(\mathrm{p}>0,05)$.

Las diferencias de PM al final de la experiencia resultaron significativas para el tratamiento aplicado (Figura 2). Los ejemplares juveniles DI presentaron mayores PM por individuo, comparados con DIII que pre- 
sentó menor PM individual, siendo significativamente distintos $(p<0,05)$. Por su parte, la DII no se diferenció significativamente de ninguna de las anteriores.

Los valores de BF obtenidos en DIII fueron superiores a DI $(\mathrm{p}<0,05)$, al tiempo que DII, presentó valores intermedios, sin diferenciarse de ninguno de los anteriores ( $>00,05)$ (Figura 3).

$\mathrm{El}$ análisis del efecto lineal de la densidad fue significativo sobre el PM, el CCE y la BF $(p<0,05)$, al tiempo que el análisis de regresión permitió determinar que las dos primeras variables se relacionaron de manera negativa con el aumento de la densidad, mientras que la BF lo hizo de manera positiva (Figuras 1, 2 y 3). La tasa de supervivencia no presentó diferencias significativas entre los distintos tratamientos ( $p>0,05)$ (Figura 4).

En un sistema de cultivo determinado, el efecto de la densidad de cría es uno de los factores más importantes a considerar sobre los parámetros de crecimiento, supervivencia y comportamiento ${ }^{5,22}$. Los resultados del presente ensayo evidencian que la densidad de cría no afecta al porcentaje de supervivencia, aunque sí afecta negativamente al PM y al CCE en ejemplares juveniles de $R$. quelen bajo un sistema de cría semiintensivo. Estos resultados coinciden con los reporta-

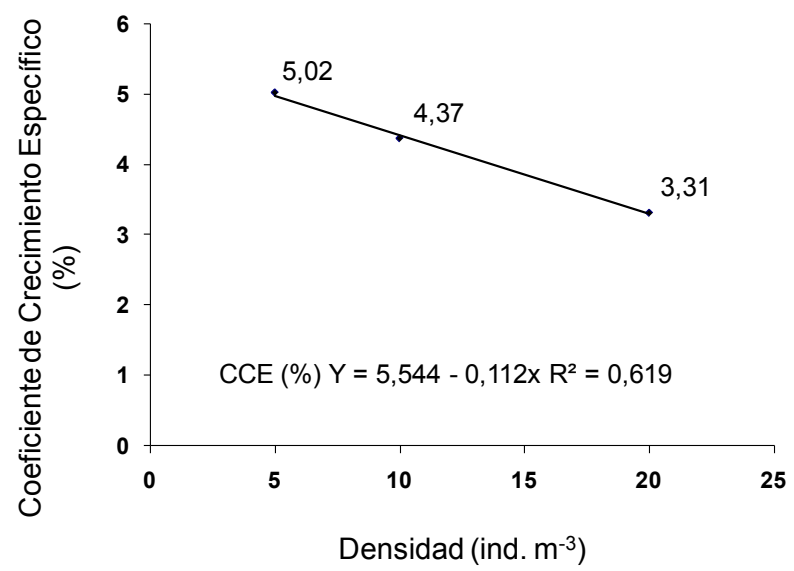

Figura 1. Coeficiente de crecimiento específico estimado al final del ensayo.

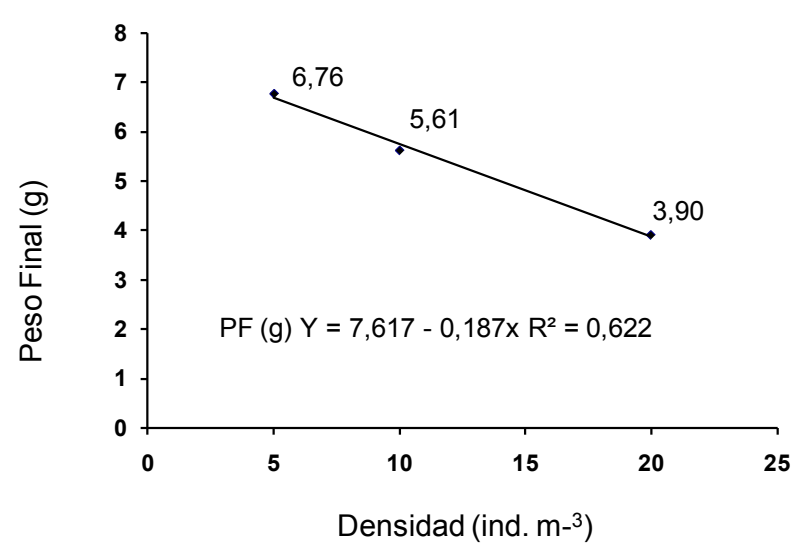

Figura 2. Peso final estimado en ejemplares juveniles de $R$. quelen criados a diferentes densidades. dos en ejemplares de Brycon cephalus ${ }^{11} \mathrm{y}$ en juveniles de $R$. quelen ${ }^{26}$ criados a distintas densidades, donde se encontró que el aumento de la densidad produce disminución del crecimiento, sin afectar las tasas de supervivencia.

En una experiencia realizada por investigadores brasileros ${ }^{2}$ con $R$. quelen juveniles criados en jaulas suspendidas a densidades de 100,200 y 300 peces $/ \mathrm{m}^{3}$, se verificó que los mejores parámetros de crecimiento (ganancia de peso, ganancia de peso diario y tasa de crecimiento específico) fueron obtenidos con la menor densidad, mientras que los porcentajes de supervivencia no se diferenciaron estadísticamente, siendo similares o los encontrados en el presente trabajo.

Resultados contrastantes fueron reportados en ejemplares juveniles de $R$. quelen criados a densidades mayores a las utilizadas en el presente estudio, donde manifestaron una correlación positiva entre el aumento de la densidad y los parámetros de crecimiento y supervivencia ${ }^{22}$. Estos factores pudieron verse favorecidos, en parte, debido a la disminución de la agresión y formación de cardúmenes al momento de alimentarse, como respuesta al aumento de las densidades, tal como lo observado en ejemplares juveniles de Clarias gariepinus ${ }^{12}$.

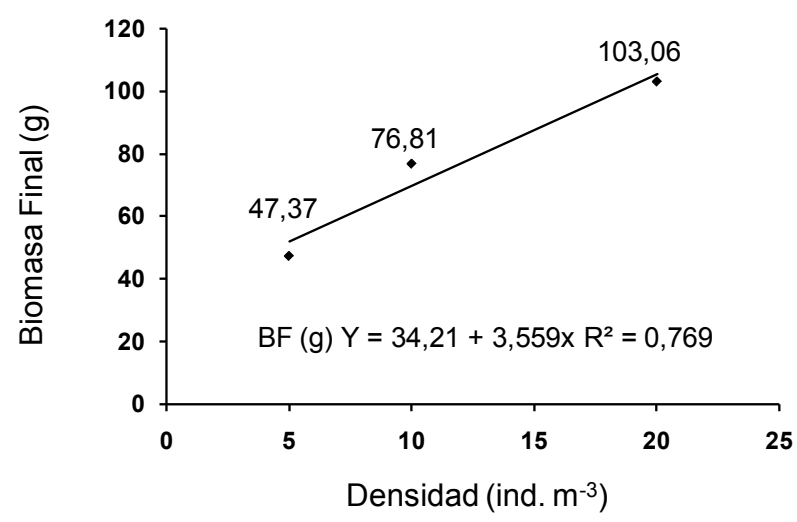

Figura 3. Biomasa final en ejemplares juveniles de $R$. quelen criados a diferentes densidades.

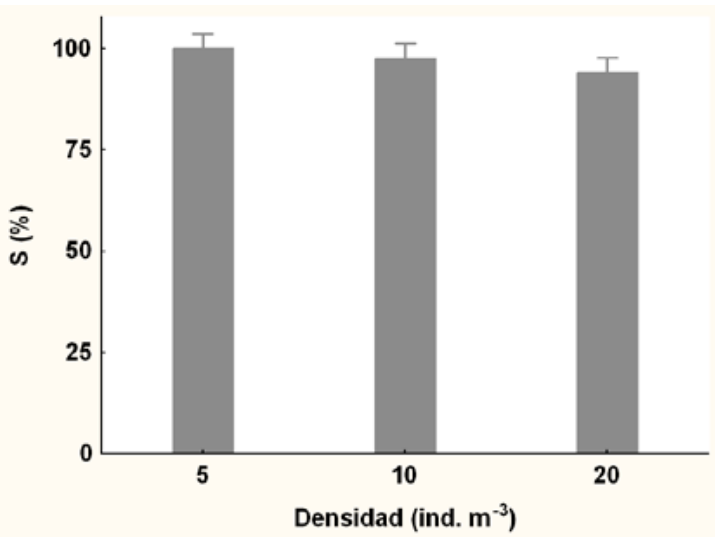

Figura 4. Supervivencia final de $R$. quelen expresada en intervalos de confianza del $95 \%$. Las diferencias fueron no significativas $(\mathrm{p}<0.05)$. 
Sin embargo, en $C$. gariepinus juveniles criados a altas densidades $\left(90,180\right.$ y 270 peces $\left./ \mathrm{m}^{3}\right)$, los parámetros de crecimiento y supervivencia estuvieron inversamente relacionados con la densidad de stock, sin presentar diferencias estadísticas significativas entre las distintas densidades ${ }^{15}$. Estudios realizados en juveniles de Heterobranchus longifilis empleando diferentes densidades de cría, demostraron que todas las variables estudiadas resultaron influenciadas por la densidad, observando que en las menores densidades se alcanzaron los valores superiores de pesos finales, ganancia de peso medio diario y tasa de supervivencia y, a su vez, las menores tasas de canibalismo, coeficiente de variación de peso y tasa de conversión alimenticia ${ }^{9}$.

Otros autores, trabajando con ejemplares juveniles de Argyrosomus japonicus a densidades de 4,08; 8,16 y $16,32 \mathrm{~kg} / \mathrm{m}^{3}$, obtuvieron mejores desempeños de crecimiento en los tratamientos de media y alta densidad, al tiempo que la tasa de supervivencia no se diferenció estadísticamente en ninguno de los tratamientos estudiados ${ }^{23}$.

En el presente estudio se observó que el porcentaje de supervivencia no se vio afectado por efecto de las diferentes densidades de stock. Estos resultados concuerdan con lo reportado por investigadores que trabajando con juveniles de $R$. quelen criados a similares densidades, obtuvieron porcentajes de supervivencia superiores al $70 \%{ }^{17,26}$. Por el contrario, peces juveniles de la misma especie criados bajo sistema intensivo evidenciaron un aumento de la tasa de supervivencia con el aumento de la densidad ${ }^{22}$.

Los resultados del presente trabajo demuestran que la densidad de 10 individuos $/ \mathrm{m}^{3}$ sería la recomendada, ya que produce aceptables valores de crecimiento y altas tasas de supervivencia. Por otro lado, si lo que se desea es obtener ejemplares juveniles con mayores pesos individuales, la densidad de siembra de 5 individuos $/ \mathrm{m}^{3}$ sería la adecuada; en cambio, si lo que se pretende es obtener un mayor número de ejemplares y por ende una mayor biomasa, el tratamiento con la mayor densidad se ajusta a este requerimiento, ya que aumenta hasta cuatro veces el número de ejemplares por unidad experimental con altos porcentajes de supervivencia. Experimentos adicionales deben ser realizados para verificar si se puede incrementar más la densidad sin reducir el crecimiento ni afectar la supervivencia de los peces.

Agradecimientos. Esta investigación fue financiada por la Agencia Nacional de Promoción Científica y Tecnológica, Proyecto BID 1728/OC-AR PICTO-UNNE 161.

\section{REFERENCIAS}

1. Baldisserotto B, Radünz J. 2004. Criação de jundiá, Ed UFSM, Santa María (Brasil), 232 p.

2. Barcellos LJ, Kreutz LC, Quevedo RM, Fioreze I, Cericato L, Benck A, Fagundes M, Conrad J, Krammer R, Bruschi A, Ritter F. 2004. Nursery rearing of jun- diá, Rhamdia quelen (Quoy \& Gaimard) in cages: cage type, stocking density and stress response to confinement. Aquaculture 232: 383-394.

3. Becker AG, Oliveira L, Kochhann D, Gonçalves JF, Loro VL, Baldisserotto B. 2009. Dissolved oxygen and ammonia levels in water that affect plasma ionic content and gallbladder bile in silver catfish. Ciência Rural 39: 1768-1773.

4. Behr ER, Tronco AP, Radünz J. 2000. Ação do tempo e da forma de suplementação alimentar com Artemia franciscana sobre a sobrevivência e o crescimento de larvas de jundiá. Ciência Rural 30: 503-507.

5. Boujard T, Labbé L, Aupérin B. 2002. Feeding behaviour, energy expenditure and growth of rainbow trout in relation to stocking density and food accessibility. Aquac Res 33: 1233-1242.

6. Cardoso AP, Radünz J, Medeiros TS, Knöpker MA, Lazzari R. 2004. Criação de larvas de jundiá (Rhamdia quelen) alimentadas com rações granuladas contendo fígados ou hidrolisados. Acta Scientiarum 26: 457-462.

7. Carneiro PC, Mikos JD, Schorer M, Oliveira PR, Bendhack F. 2003. Live and formulated diet evaluation through initial growth and survival of jundiá larvae, Rhamdia quelen. Scientia Agricola 60: 615-619.

8. Carneiro PC, Mikos JD. 2005. Freqüência alimentar e crescimento de alevinos de jundiá, Rhamdia quelen. Ciência Rural 35: 187-191.

9. Coulibaly A, Ouattara IN, Koné T, N'Douba V, Snoeks J, Gooré G, Kouamélan EP. 2007. First results of floating cage culture of the African catfish Heterobranchus longifilis Valenciennes, 1840: Effect of stocking density on survival and growth rates. Aquaculture 263: 61-67.

10. FAO. 2010. El estado mundial de la pesca y la acuicultura, Publ. Departamento de Pesca y Acuicultura de la FAO, Roma, 219 p. On line: http://www.fao.org.

11. Gomes LC, Baldisserotto B, Senhorini JA. 2000. Effect of stocking density on water quality, survival, and growth of larvae of the matrinxã, Brycon cephalus (Characidae), in ponds. Aquaculture 183: 73-81.

12. Hecht T, Uys W. 1997. Effect of density on the feeding and aggressive behaviour in juvenile African catfish, Clarias gariepinus. S Afr J Sci 93: 537-541.

13. Hepher, B. 1988. Nutrición de peces comerciales en estanques, Limusa, México, $406 \mathrm{p}$.

14. Hernández DR, Sánchez S, Santinón JJ, Domitrovic HA. 2009. Fontes não-convencionais de proteína na primeira alimentação do bagre sul americano (Rhamdia quelen). Ciência Rural 39: 878-884.

15. Jamabo NA, Keremah RI. 2009. Effects of stocking density on the growth and survival of the fingerlings of Clarias gariepinus (Burchell, 1822). J Fish Int 4: 55-57.

16. Jomori RK, Carneiro DJ, Espagnoli MI, Martins G, Portella MC. 2005. Economic evaluation of Piaractus mesopotamicus juvenile production in different rearing systems. Aquaculture 243: 175-183.

17. Luchini L, Avendaño T. 1985. Pond culture experiments of South American catfish, Rhamdia sapo, fingerlings. Prog Fish-Cult 47: 241-243. 
18. Luchini L, Quiros R. 1990. Cage culture of South American catfish (Rhamdia sapo) Preliminary results in the Salto Grande reservoir (Argentina). J Aqua Trop 5: 163-172.

19. Luchini L, Panné S. 2008. Perspectivas en acuicultura: nivel mundial, regional y local. Publ. Dirección de Acuicultura, Subsecretaría de Pesca y Acuicultura, SAGPyA, Buenos Aires, Argentina, 98 p.

20. Marchioro MI, Baldisserotto B. 1999. Sobrevivência de alevinos de jundiá (Rhamdia quelen Quoy \& Gaimard, 1824) à variação de salinidade da água. Ciência Rural 29: 315-318.

21. Piaia R, Radünz J. 1997. Avaliação de diferentes fontes protéicas sobre o desempenho inicial de larvas do jundiá Rhamdia quelen. Ciência Rural 27: 319-323.

22. Piaia R, Baldisserotto B. 2000. Densidade de estocagem e crescimento de alevinos de jundiá Rhamdia quelen (Quoy \& Gaimard, 1824). Ciência Rural 30: 509-513.

23. Pirozzi I, Booth MA, Pankhurst PM. 2009. The effect of stocking density and repeated handling on the growth of juvenile mulloway, Argyrosomus japonicus (Temminck \& Schlegel 1843). Aquacult Int 17: 199-205.
24. Rossi F, Luchini L. 2005. Tecnologías para cultivo del "randia" (Rhamdia quelen) para fomento de su producción comercial, en clima templado a templado-cálido, monografía, Sitio Argentino de Producción Animal. On line: http://www.produccion-animal.com.ar/produccion_peces/ piscicultura/09-randia_sagpya.pdf.

25. Salhi M, Bessonart M, Chediak G, Bellagamba M, Carnevia D. 2004. Growth, feed utilization and body composition of black catfish, Rhamdia quelen, fry fed diets containing different protein and energy levels. Aquaculture 231: 435-444.

26. Sánchez S, Santinón JJ, Hernández DR, Roux JP, Domitrovic HA. 2008. Cría de bagre sudamericano (Rhamdia quelen) en estanques luego de diferentes períodos de retención del crecimiento a tres densidades de siembra. Rev electrón vet 9: 1-9.

27. Silvergrip, AM. 1996. A systematic revision of the Neotropical catfish genus Rhamdia (Teleostei, Pimelodidae). PhD Thesis, Department of Zoology, University of Stockholm, Sweden, $156 \mathrm{p}$. 